University of New Mexico

UNM Digital Repository

6-1-1977

\title{
Optimal foraging: A selective review of theory and tests
}

\author{
G.H. Pyke \\ H.R. Pulliam \\ Eric Charnov
}

Follow this and additional works at: https://digitalrepository.unm.edu/biol_fsp

Part of the Biology Commons

\section{Recommended Citation}

Pyke, G.H., H.R. Pulliam and E.L. Charnov. 1977. Optimal foraging: A selective review of theory and tests. Quarterly Review of Biology 52:137-154

This Article is brought to you for free and open access by the Academic Department Resources at UNM Digital Repository. It has been accepted for inclusion in Biology Faculty \& Staff Publications by an authorized administrator of UNM Digital Repository. For more information, please contact disc@unm.edu. 
vOL. 52 , NO. 2

June, 1977

\title{
The Quarterly Review of B IOLOGY
}

\section{OPTIMAL FORAGING: A SELECTIVE REVIEW OF THEORY AND TESTS}

\author{
By G. H. Pyke,* H. R. Pulliam, $†$ and E. L. Charnov* \\ *Department of Biology, University of Utah, Salt Lake City, Utah 84112 \\ $\dagger$ Department of Ecology and Evolutionary Biology, University of Arizona, \\ Tucson, Arizona 85721
}

ABSTRACT

Beginning with Emlen (1966) and MacArthur and Pianka (1966) and extending through the last ten years, several authors have sought to predict the foraging behavior of animals by means of mathematical models. These models are very similar, in that they all assume that the fitness of a foraging animal is a function of the efficiency of foraging measured in terms of some "currency" (Schoener, 1971) - usually energy - and that natural selection has resulted in animals that forage so as to maximize this fitness. As a result of these similarities, the models have become known as "optimal foraging models"; and the theory that embodies them, "optimal foraging theory."

The situations to which optimal foraging theory has been applied, with the exception of a few recent studies, can be divided into the following four categories: (1) choice by an animal of which food types to eat (i.e., optimal diet); (2) choice of which patch type to feed in (i.e., optimal patch choice); (3) optimal allocation of time to different patches; and (4) optimal patterns and speed of movements. In this review we discuss each of these categories separately, dealing with both the theoretical developments and the data that permit tests of the predictions. The review is selective in the sense that we emphasize studies that either develop testable predictions or that attempt to test such predictions in a precise quantitative manner. We also discuss what we see to be some of the future developments in the area of optimal foraging theory and how this theory can be related to other areas of biology.

Our general conclusion is that the simple models so far formulated are supported reasonably well by available data and that we are optimistic about the value both now and in the future of optimal foraging theory. We argue, however, that these simple models will require much modification, especially to deal with situations that either cannot easily be put into one or another of the above four categories or entail currencies more complicated than just energy. 
INTRODUCTION

A LL ANIMALS require food. Some must actively search, pursue, capture, and consume many prey in order to survive and leave offspring. A comparatively new body of literature, which began with Emlen (1966) and MacArthur and Pianka (1966), and now known as optimal foraging theory (Schoener, 1971; Pulliam, 1974; Pearson, 1974, 1976; Werner and Hall, 1974; Westoby, 1974; Charnov, 1976a,b; Covich, 1976), has attempted to explain and predict many aspects of the foraging behavior of animals. Much of this theory assumes that the fitness associated with an animal's foraging behavior has been maximized by natural selection, subject to certain constraints. The selection that has so far been considered, either implicitly or explicitly, is Darwinian natural selection coupled with genetic inheritance, but evolution could also be cultural and yet be governed by selection. In either case the basic argument runs as follows:

(1) Behavior in general, and foraging behavior in particular, show heritable variation; and this entails variation in the contribution to subsequent generations.

(2) There is a range of possible foraging behaviors. In other words, there are constraints in the system. For example, an animal may or may not be able to alter its rate of encounter with a particular food type by altering its own behavior.

(3) Natural selection will favor those individuals in a population which contribute the most to subsequent generations. Hence, natural selection will result in a change with time of the average foraging behavior in the populations, towards that foraging behavior in the range of possible behaviors which gives maximum fitness. It is assumed (usually implicitly) that the rate with which these changes occur is much greater than the rate with which the position of the maximum fitness behavior changes. From this it follows that the average foraging behavior in a population should be very close to the behavior which results in maximum fitness, subject to whatever constraints are operating. This postulate forms the basis for hypotheses concerning how animals forage in particular circumstances.

To formulate a precise hypothesis about foraging behavior while using the above basis as a starting point, it is necessary to determine the relationship between fitness and variations in the foraging behavior, and to determine the range of possible foraging behavior. For example, in considering an animal's diet, one might assume that fitness is an increasing function of the net rate of energy gain and that after encountering a certain food type, an animal can choose either to eat it or to ignore it. In this case it would be hypothesized that the animal's pattern of choice of food type (i.e., its diet) will be such that the net rate of energy intake is maximized. In general, the hypothesized foraging behavior will always be such that something is maximized or minimized, and hence the term optimal foraging theory is appropriate for the resulting body of theory. Schoener (1971) reviewed the subject (see Krebs, 1974; Rapport and Turner, 1977) and characterized the procedure for finding the optimal behavior as having three parts: (1) choosing a currency, (2) choosing the appropriate cost-benefit functions (establishing the constraints), and (3) solving for the optimum. The currency in this case is energy, the cost-benefit function is the mathematically derived relat nship between the animal's diet and its net rate of energy intake (see section on optimal diet), and the optimum will be found by determining the diet which maximizes the net rate of energy intake subject to the various constraints. In general, there is no recipe for determining just what the currency and constraints should be in a particular situation, and it will always be the job of the naturalist to understand the biology of an animal sufficiently well to know which currency is being optimized.

In almost all optimal foraging studies to date the currency has been assumed to be the net rate of energy intake, and the basic hypothesis has been that this intake rate will be maximized [for exceptions, see Rapport (1971), Covich (1972, 1976), Janzen and Freeland (1974), Westoby (1974), Gill and Wolf (1975a, b), Pulliam (1975), Thompson, Vertinsky, and Krebs (1975), Wolf (1975), Wolf, Hainsworth, and Gil (1975), and DeBenedictus et al. (in press)]. Before proceeding, it seems appropriate to review the arguments in favor of adopting such a currency (see Schoener, 1971). First, if an animal has a fixed energy requirement, if it gains nothing in terms of fitness from a greater amount of 
energy, and if it requires time to perform other activities, then the fitness of the animal should be greatest when it minimizes the time required to obtain the fixed amount of energy and uses the time left over to perform the other activities. In this case the net rate of energy intake while the animal is foraging would be maximized, and such animals have been referred to as time minimizers by Schoener (1971). Secondly, if an animal has a fixed amount of time in which to forage and if fitness increases continually with increasing amounts of energy obtained, then the maximum fitness will occur when the animal obtains the maximum amount of energy in the allotted time. Such animals have been referred to as energy maximizers by Schoener (1971); in this case too, the net rate of energy intake while foraging is maximized.

Whether or not an animal falls into one of these two categories depends on whether or not other factors, such as predation or nutritional requirements, operate at the same time and behave in a way that produces a conflict with increasing the net rate of energy (or food) intake. For example, if the habitat patch that is best in terms of net rate of energy intake is extremely poor in terms of having a high density of predators, then an animal might not be expected to feed in that patch. On the other hand, predation will not affect optimal diet if the choice of one or another food type does not affect the probability of being eaten by a predator, even though both foraging and predation occur simultaneously. To decide whether these factors, such as predation, nutritional requirements, mate search, and aggressive interactions, need to be included in the analyses, it will be necessary to rely on detailed knowledge of an animal's biology. Since all animals require energy, it should almost always be included in an optimal foraging analysis; and if there is no conflict with other factors, animals should be either time-minimizers or energy- (or food-) maximizers and hence should forage in such a way that their net rate of intake of energy (or food) is maximized (Schoener, 1971).

The extent to which additional factors, such as predation and nutrtion, have to be included in the optimal foraging analyses has not been established. Schoener (1971) has argued that these factors, in general, will be unimportant. Only one author (Pyke, 1974, in press-a), however, has argued from the biology of the study animal that the most realistic currency is in fact the net rate of energy intake and that other factors are not important. Belovsky (unpub.) has quantitatively included dietary constraints. In all other studies, in which the currency used is net rate of energy intake, the use is justified solely by the assumption that it seems appropriate. This assumption is, we believe, justified in most of the studies discussed in this paper, but it should not be made blindly in every instance.

A further important consideration in choosing the appropriate currency is the time scale over which to carry out the optimization. For example, foraging behavior that maximizes the amount of energy obtained by an animal over a week or a month might be different from foraging behavior that maximizes the amount obtained in one hour or one day (Katz, 1974). Should the time scale for the maximization of currency be a day or a week, or what? Since biological fitness is measured over the entire life history of an individual, it is this length of time that is relevant. If, however, (foraging) behavior at one point in time does not alter the optimal (foraging) behavior at a later point in time, then the predictions from the optimization are independent of time scale, and hence any time scale can be used.

There are instances in which the behavior of an animal at one point in time will affect the optimal behavior at a later point in time. They fall into three classes. The first class consists of behaviors which "commit" the animal for some length of time into the future. For example, nest placement for a bird often commits the bird to feeding in the vicinity of its nest for quite a long time, and hence the location of the nest will affect the kinds and abundance of food items near the nest for the entire nesting period (Orians and Pearson, in press). The appropriate time scale for optimal nest placement would then be the length of the nesting period. However, the appropriate time scale for optimal foraging behavior of the bird during the nesting period could be much less, so long as the nest position was taken as a fixed constraint in the system.

Another example is that when an animal chooses to visit a particular habitat patch it is committing itself to spending some amount of time in that patch. In this case, the animal might be hypothesized to make the following four decisions, each one of which could easily depend 
on the other three: (1) which patch types to visit; (2) how long to stay in each patch; (3) which food types to eat in each patch type; and (4) which foraging path to employ in each patch type.

These four decisions give four categories into which most of the optimal foraging papers so far published can be placed, and we shall use these categories in later discussion of the work that has been done. The reason that these categories are so distinct is that the four decisions have so far been treated quite separately. Furthermore, in all cases this separation is based on the implicit assumption that the decisions are approximately independent.

The second class of behaviors in which what an animal does at one point in time will affect the optimal action at a later time are those which change the conditions impinging on the animal at the later time, by virtue of some direct effect of the animal on the environment-for example, when an animal is likely to return to its present position in order to forage at a later time. The abundance of food at this position at the later time will be a function of the behavior of the animal in question at the present time and of the behaviors of other animals which may visit the same position in the intervening time. The extent to which the behavior of the animal in question will affect the abundance of food at a later time will therefore depend, in part, on the extent to which other animals are likely to visit the same positions-i.e., on the extent to which the animal has exclusive use of an area. Hence, in general, this class of behavior is most likely to occur when animals have some degree of exclusive use of an area. If an animal has such exclusive use of an area, then it could "manage" its resources for "sustained yield" rather than maximize the initial yield at the cost of poorer yields later (Charnov, 1973; Charnov, Orians, and Hyatt, 1976). As yet, no published studies deal with this possible phenomenon.

The third class consists of behaviors which provide the animal with information about changing spatial-temporal distributions of resources. If the distribution of a resource does in fact change in space and time it may well be advantageous for an animal to spend time "exploring" or "being curious" to the detriment of immediate foraging efficiency, if the information so gained will enable it to switch its behavior rapidly as conditions change (Royama,
1970). A possible example of this is discussed in the section on optimal patch choice.

Given that we can find the appropriate currency and solve the cost-benefit function to get the optimal foraging strategies, where does it all lead? The place of foraging theory as a tool for predicting ecological phenomena other than the foraging process itself was first explored by MacArthur and Pianka (1966), who predicted the changes in consumer choice occurring as the number of competing species increases. Their conclusions, now referred to as the "compression hypothesis" (MacArthur and Wilson, 1967) are that as the number of competing species increases (1) the variety of habitats used should contract, and (2) the range of food items taken within habitats should remain constant or increase slightly. As reviewed by MacArthur (1972) in Geographical Ecology, the compression hypothesis is a simple idea formulated to predict foraging behavior as a tool for understanding competitive relationships among organisms (see also Schoener, 1974). A rather different approach was taken by Werner (in press), whose work we will discuss in more detail (see below, p. 151).

In this paper we attempt to review briefly the theory and experimental results pertaining to the four categories of foraging mentioned above, namely, optimal diet, patch choice, allocation of time to patches, and the foraging path. These are the elements of a "microecological theory," in the sense that the foraging path, patch, and diet choice, combined with the distribution of prey in space and time, determine the small-scale events, the sum of which is the very core of such large-scale population phenomena as predation and competition. Thus, we view foraging theory as the heart of a microecological theory that forms a basis of a "macroecological theory" of the dynamics of populations. This is not a break with traditional population modeling, but rather a supplement to it where such usually static parameters as niche width, predation coefficients, and competition coefficients can be modeled as dynamic terms that change according to varying availability of food. For the sake of brevity, we restrict our attention, for the most part, to papers which test predictions arising from optimal foraging models in a direct and quantitative manner. Hence, this is to be a selective rather than a comprehensive review. 
OPTIMAL DIET

The nature of food choice has received attention from many authors (see Charnov, 1976). Most studies have been theoretical, and only a few have attempted precise tests of the theories (Werner and Hall, 1974; Menge and Menge, 1974; Emlen and Emlen, 1975; Charnov, 1976a; Goss Custard, in press; Krebs, Erickson, Webber, and Charnov, in press; Belovsky, unpub.).

Theories in this area and their derivations are very similar from one author to another, and one basic result has been independently derived by no less than nine authors (Schoener, 1969, 1971; MacArthur, 1972; Charnov, 1973, 1976a; Timin, 1973; Maynard Smith, 1974; Pearson, 1974; Pulliam, 1974; Werner and Hall, 1974; Estabrook and Dunham, 1976). We briefly describe this result here before proceeding to individual papers. The result is that the diet which maximizes the net rate of energy or mass intake (i.e., the optimal diet) in a fine-grain situation is obtained as follows:

(1) Each food type has an associated food value measured in calories or units of weight and an associated handling time. Food types are first ranked by their ratios of the food value to handling time.

(2) In addition to time spent handling food items, time must be spent searching for them. These times are assumed to be mutually exclusive and to account for all the time available for foraging. Taking into account the handling times, food values, and search time (which is a function of the diet of the animal), it is then possible to calculate the net rate of food gain for any possible diet.

(3) Finally, the optimal diet is determined by beginning with the highest value of the ratios of food value to handling time, and then adding food types to the diet in their rank order. This process is continued so long as the ratio of food value to handling time for each addition to the diet is greater than the net rate of food intake for the diet without the addition. When this inequality reverses, the optimal diet has been obtained. For a detailed mathematical discussion of this result, see Charnov (1976a).

This optimal diet has three properties which should be noted. The first of these is that whether or not a food type should be eaten is independent of the abundance of that food type and depends only on the absolute abun- dances of the food types of higher rank. So, in theory, an animal should never specialize on a "less preferred" food type regardless of its abundance. Estabrook and Dunham (1976) have claimed that both relative and absolute abundances of food types are important, but, in their analysis, relative and absolute abundances were not independent. The second property is that, in general, as the abundance of a preferred food type included in the diet increases, the number of less preferred food types included in the optimal diet will shrink. In other words, increasing food abundance should lead to greater food specialization. And, in particular, as the abundance of an item included in the optimal diet becomes infinitely large, all food items of lower rank are dropped out of the diet. If the diet of an animal does change, then items should be added to or dropped from the diet in their rank order [see (3) above]. The third property is that a food type is either completely included in the optimal diet or completely excluded from it. In other words, if a food type is included it should be eaten whenever encountered, and if excluded it should never be consumed. Hence, animals should never exhibit "partial preferences." However, if the theory is extended to include dietary constraints or random variations in the food abundances, then partial preferences are to be expected (Westoby, 1974; Pulliam, 1975; Pearson, 1976; Rapport, 1971; Rapport and Turner, 1977; Altmann and Wagner, 1977).

Of the three predicted properties of an optimal diet, only the most qualitative one was supported prior to experiments by Werner and Hall (1974), Emlen and Emlen (1975), Charnov (1976a), Goss Custard, 1976, and Krebs, et al. (in press). This is the prediction that increasing food abundance leads to greater food specialization. Ivlev (1961) first demonstrated this with laboratory experiments on various species of fish. For a review of papers containing data that support this simple idea, see Schoener (1971); and for a more recent review of such data from fish, see Eggers (1975). The data in these papers cannot be used to test precisely the optimal-diet predictions because no information is given about food, energy values, or handling times of the various food types. Several recent studies have shown that animals select prey of high profitability (e.g., Kear, 1962; Smith, 1970; Menge and Menge, 1974). 
We are now in a position to consider, in greater detail, the work mentioned above (Werner and Hall, 1974; Charnov, 1976; Goss Custard, 1976; and Krebs et al., in press). One prediction these papers test is that the optimal diet always includes the food type of highest rank, and food types are added to the diet in rank order as food abundance decreases. However, this prediction, as tested by Charnov (1976a), is only a qualitative one about the order in which food items are added to the diet. The other three papers have tested the quantitative prediction that the position in a foodabundance continuum, at which each food item is added to or dropped from the diet, is given by the criteria developed from a mathematical formulation of the above description of the optimal diet.

Charnov (1976a) analyzed data obtained by C. S. Holling on mantids which were offered houseflies as food. These houseflies, which were all of the same size, were offered at different distances from the mantids. Charnov showed that these different distances determine a ranking of food types that corresponds to the ratio of food value to handling time. In this case, the ratio decreases as the distance at which a fly is offered increases. Charnov further shows that the amount of food in the gut of a mantid enables the mantid to estimate its net rate of food gain at any point in time and hence to estimate the overall abundance of food. The prediction, then, is that the lower the level of food in the gut of a mantid, the further it should pursue or stalk for houseflies. In other words, as the overall abundance of food de- creases, the mantid should include in its diet flies at greater and greater distances. When the maximum reaction distance of the mantids is plotted against the amount of food in the gut, a negative relationship is found, as predicted. Charnov points out, however, that it is not possible to rule out alternative explanations for this result. The most plausible of these is that the stalking of prey entails a risk of predation which increases with the distance over which the mantid must stalk. This risk would lead to a tendency on the mantid's part not to stalk prey at great distances, and this tendency would be expected to decline as the importance of obtaining food increased (i.e., as the amount of food in the gut decreased).

Werner and Hall (1974) examined diets of bluegill sunfish allowed to feed on daphnia of three different size classes. As the parameter of food abundance they used the mean time between encounters with the largest sized daphnia $\left(T_{s}\right)$; hence the prediction they tested was that as $T_{s}$ increased the diet should increase and include more and more of the smaller size classes, and also that particular size classes should be dropped from the diet at predictable values of $T_{s}$. For each predicted diet they calculated expected relative proportions of different size classes in the diets of the fish, and compared these with the observed. Observed diets are impressively similar to those predicted (Table 1). The only departure from prediction occurred when $T_{s}$ was less than 29 seconds, and then the fish ate more of Size Class 2 than expected.

Krebs et al. (in press) tested the prediction that whether or not a prey type is eaten is inde-

TABLE 1

Comparison of observed and expected diets of bluegill sunfish (Adapted from Werner and Hall, 1974)

\begin{tabular}{llccc}
\hline & & \multicolumn{2}{c}{ EXPECTED/OBSERVED } \\
RANGE OF $\mathrm{T}_{\mathrm{S}}$ & \multicolumn{1}{c}{ OPTIMAL DIET } & $\begin{array}{c}\text { Size } \\
\text { Class } 1\end{array}$ & $\begin{array}{c}\text { Size } \\
\text { Class 2 }\end{array}$ & Class 3 \\
\hline $\mathrm{T}_{\mathrm{S}}>295 \mathrm{sec}$ & $\begin{array}{l}\text { All size classes } \\
\text { eaten as encountered }\end{array}$ & $1.0 / 1.0$ & $1.0 / 0.9$ & $1.0 / 0.9$ \\
$29 \mathrm{~s}<\mathrm{T}_{\mathrm{S}}<295 \mathrm{~s}$ & $\begin{array}{l}\text { Two largest size classes } \\
\text { should be eaten }\end{array}$ & $1.0 / 1.0$ & $0.71 / 0.58$ & $0.0 / 0.04$ \\
$\mathrm{~T}_{\mathrm{S}}<29 \mathrm{~s}$ & $\begin{array}{l}\text { Only largest size class } \\
\text { should be eaten }\end{array}$ & $1.0 / 1.0$ & $0.0 / 0.23$ & $0.0 / 0.05$ \\
\hline
\end{tabular}

$T_{S}$, mean time between encounters with the largest sized daphnia. 
pendent of its own abundance (and depends only on the abundance of prey of higher rank). Using Great Tits (Parus major), foraging for parts of meal worms presented on a moving conveyor belt, they found the above prediction to be supported over a fairly wide range of prey availability (for two types of prey - meal worms of two sizes). Not supported is the prediction that a certain availability for the first-ranked prey, the second-ranked is completely dropped from the diet. Their work showed, instead, that the second-ranked prey is dropped gradually from the diet, rather than in the abrupt step predicted. Pearson (1976) should be consulted for a theory predicting this gradual change.

Goss Custard (in press) has provided the best field evidence to date for optimal prey choice. In a field study, he found that Redshank preferred the size classes of Nereis diversicolor and Nephtys hombergi with the highest reward per unit handling time (large prey), and that this preference was dependent on the encounter with large, but not with small, prey. He incorporated the observed preference relationships into a simulation model and was able to show that the Redshank selects the optimal diet.

The successes of these studies in predicting an animal's diet from theoretical arguments is certainly an encouraging beginning, but many more studies of a similar kind are needed before any generality can be reached. Fur thermore, we expect that such success for the simple diet theories will only be forthcoming in rather special circumstances. Only when animals are foraging for a source of energy such as plant nectar, or for different-sized items of one kind of food, will the simple theories be predictive. However, in such cases as herbivores foraging on a variety of plants, each one of which contains toxic compounds, the effects of which depend on the amounts ingested and the interactions between the compounds (and so on), so simple a theory seems doomed to failure (Westoby, 1974; Janzen and Freeland, 1974; Belovsky, unpub.). Clearly, a theory that includes elaborate nutrient constraints will be needed, and the present simple explorations in this direction will probably prove too simple (e.g., Pulliam, 1975).

An example of a study which includes various constraints is the recent work of Belovsky (unpub.). He used linear programming to determine the optimal diet of a moose which was assumed to feed on three food types (aquatic vegetation, forbs, and foliage) while being subject to a number of constraints. One constraint was that the moose must have a net daily energy gain that is non-negative. Another constraint was that thermoregulatory requirements placed upper limits on the amounts of time that the moose could spend feeding in the aquatic and terrestrial habitats respectively. A third constraint was that the moose must achieve a certain minimum daily intake of sodium. These and other constraints were expressed as linear functions of the amounts eaten of the three food types. Belovsky (unpub.) derived the optimal diets, in terms of daily intake of the three food types, under two hypotheses and compared the observed diets with the expected optimal diets. The two hypotheses were: (1) a moose attempts to consume the greatest amount of energy per day, subject to the constraints, and (2) a moose attempts to satisfy its daily energy requirements in the least amount of foraging time, subject to the constraints. Belovsky (unpub.) finds that the predicted diets under both these hypotheses are fairly close to the observed diets in different situations but concludes that the observed diets agree best with the energy maximization hypothesis. Belovsky (unpub.) also argued that the model could predict the optimal size for an adult moose. Interestingly, there was a major deviation between the predicted and observed relative sizes of a bull versus a cow. The model predicted a bull to be smaller than a cow, while the reverse is actually observed. It appears that the mating advantage to a bull of being large overrides, in terms of fitness, the energetic considerations of size (Belovsky, unpub.). This conclusion indicates that the choice of the appropriate currency is all-important!

Emlen and Emlen (1975) also have provided a quantitative test of a slightly modified form of the optimal-diet theory discussed above. They considered two types of seeds with a frequency distribution of size within each type and calculated the proportions of the two seed types in the optimal diets of lab mice. These proportions were converted into expected numbers of seeds eaten and then compared with the observed numbers eaten. The observed and expected (i.e., optimal) numbers appeared to be quite similar (see Table 2 of Emlen and Emlen, 1975), but statistically significant differences 
existed in about half the cases considered. Several plausible reasons for this discrepancy between observed and predicted were proposed (Emlen and Emlen, 1975).

Before leaving the consideration of the optimal diet, the work of D. S. Wilson (in press) should be mentioned. Wilson did not actually test optimal diet predictions; instead, he assumed them to be qualitatively true and then looked at several consequences of this assumption. His purpose was to compare tropical versus temperate bird communities with respect to food availability. Recall that the method for finding an optimal diet first included a ranking of food items according to the ratio of energy over handling time. Schoener (1971) and Werner (1974) have argued (and produced supporting data) that for prey of relatively small size the order of rank should be the same as the order of size. If this is so, then the the smallest items actually in the diet should reflect the cutoff point (i.e., items of lower rank should be passed over) and thus reflect also the average rate of food intake during a foraging bout. Two field studies have correlated minimum food size with energy available qualitatively for birds (Lack and Owen, 1955; Ward, 1965). Several studies demonstrate a total or nearly total neglect of items below a certain size for birds, lizards, and fish (Root, 1967; Andrews, 1971; Ware, 1971; Sexton Baumen, and Owen, 1972).

D. S. Wilson (in press) analyzed the stomach contents from various tropical (Puerto Rico, Panama) and temperate (Colorado) bird species. Defining the minimum acceptable food size as the smallest size class containing 0.05 per cent or more numerically of the total gut contents, he showed that (1) the minimum acceptable food size in Colorado was smaller in less productive winter months than during the more productive summer; and (2) that in the tropics it was relatively constant between seasons but smaller than in Colorado. The latter observation clearly suggests a lower availability of energy in the tropics. Wilson's method corrected for the size of the birds. Furthermore, the patterns observed can probably not be explained by the distribution of prey size in the field - most studies have shown that small insects are relatively more abundant in the temperate zones (Schoener and Janzen, 1968). Thus Wilson (in press) has used optimal foraging theory to gain insight into other related phenomena.

\section{OPTIMAL PATCH CHOICE}

The problem of which patches an animal should forage in is formally analogous to the optimal diet problem only if each patch must be encountered before it is accepted or rejected. To date there are no studies of this kind of foraging. If an animal can learn the locations and types of patches in an area, however, a patch can be accepted or rejected before it is encountered. The animal may then be able to adjust the encounter rates with various patch types so that a great deal of specialization may be favored. In some situations it is most advantageous for an animal to move directly from one patch of the best type to another. This would certainly be the case if the "cost" of moving between patches were very small and if the animal knew the locations of all the best patches.

Smith and Dawkins (1971) and Smith and Sweatman (1974) have studied the behavior of titmice allowed to feed in areas of different food abundance. There were four and six such areas, respectively, and the durations of feeding bouts for the birds were limited to short periods of time so that the abundance of food would have changed only slightly. The birds had a very short distance to fly to the different areas and were probably able to learn the locations of the areas with ease. Under such conditions the optimal behavior is clearly to allocate all available time to the area of greatest food abundance. This the birds did not do. Instead, they allocated the greatest amount of time to the area of highest food abundance and progressively less time to progressively worse areas. Can this still be construed as optimal foraging? A further result obtained by Smith and Dawkins (1971) and Smith and Sweatman (1974) suggests that this behavior may be an adaptation to a fluctuating environment and that it represents a long-term adaptation rather than a short-term one (Smith and Sweatman, 1974). They found that if they changed food abundance in the four areas such that the array of abundance remained unaltered but the locations of the areas of different quality were changed, then the birds would gradually respond to the new conditions and would once again match the times spent in the areas with the abundance of food in them. The rate of this response depended on how the experiment was carried out, however. The response was quite 
rapid when the second-best area became the best area, but it was much slower when the worst area became the best. This response might well be adaptive, in the following manner (Smith and Sweatman, 1974). If the natural environment of the titmice undergoes fluctuations such that areas which have high food abundance at one time have low food abundance at a later time, and vice versa, then it would be adaptive for the birds to sample all areas continually in order to track the environment. Furthermore, if areas of lowest food abundance were less likely to become areas of high food abundance than areas of medium food abundance, then it would be advantageous to match the amount of time spent in areas with the abundance of food in the areas. This is exactly what the tits did in the experiment. In this case, the short-term adaptive strategy of allocating all the available time to the best area would not be as good as the long-term strategy of matching the time spent in different areas with the quality of the areas.

In order to test this idea further, more work, both of a theoretical and an empirical nature, will be needed. In terms of theory it will be necessary to determine the optimal pattern of allocation of time to areas of different food abundance, and this pattern should depend on the exact nature of the fluctuations of food abundance in space and time, as well as on the nature of the sampling scheme employed by an animal in assessing the quality of an area. In terms of empirical information, it will be necessary to determine the manner in which an animal samples an area and the spatial-temporal distribution of its food.

In many situations the long-term and shortterm optimal foraging strategies differ, and fitness is likely to be related most closely with the long-term outcome. An example might be any territorial animal or, in general terms, any animal which has exclusive or almost exclusive use of a foraging area. Such an animal might obtain a higher long-term yield by foraging below peak short-term efficiency. This would be particularly true for an animal which is harvesting a self-renewing resource. Then to eat all the resource might be the best short-term strategy, but it could have catastrophic long-term effects. We predict, however, that animals which do not have almost exclusive use of their foraging area will utilize the short-term strategies rather than the long-term strategies, whenever there is any competition for food between individuals. In other words, an animal would gain nothing by conserving its food for other individuals to eat.

\section{Optimal Allocation of Time to Patches}

Another problem animals face is when to leave one patch and move to another. As an animal spends more and more time in one patch it removes food from the patch and its success at finding additional food will decrease until a point is reached at which it is no longer profitable to remain in the patch. Study of this problem dates back at least as far as Gibb (1958, 1960), who put forward the "hunting by expectation" hypothesis to explain his observations on the foraging behavior of titmice. This hypothesis states simply that an animal learns to expect a certain amount of food from each patch and leaves a patch when it has obtained that amount of food. The application of optimal foraging theory to this problem is very recent, and only a few papers have so far pursued this approach (Tullock, 1970; Emlen, 1973; Krebs, Ryan and Charnov, 1974; Charnov, 1976b; Charnov, Orians and Hyatt, 1976; Cook and Hubbard, in press). We shall discuss here the work of Krebs, Ryan, and Charnov (1974), since it includes the essentials of the existing theory on the subject and is the only study which so far attempts to test the theory in a precise quantitative manner. These authors, in addition to testing the optimal foraging hypothesis, were also able to test Gibb's "hunting by expectation" (number or amount) hypothesis and a modification of his hypothesis which they call a "hunting by time expectation." Their results support only the optimal foraging hypothesis. Cook and Hubbard (in press) provide data in qualitative support of the theory.

The mathematical development of the theory in this case is analogous to that in the case of optimal diets, and the resulting prediction may be stated quite simply. It is that an animal should leave a patch when its rate of food intake in the patch drops to the average rate for the habitat, and furthermore that this "marginal" capture rate should be equalized over all patches within a habitat (Krebs, Ryan, and Charnov, 1974; Charnov, 1976b; Cook and Hubbard, in press). This prediction has been termed the "marginal value theorem" by Charnov (1976b). In order to test this prediction, how- 
ever, it is necessary to make some assumptions about the manner in which the animals estimate the quantities inherent in the theory. We shall return to this problem of estimation later. That animals can estimate the average rate of food intake for a habitat seems reasonable, but it would certainly be more difficult for them to estimate the marginal capture rates. Krebs, Ryan, and Charnov (1974) considered the foraging of tits for meal worms on artificial pine cones, and implicitly assumed that these birds have a giving-up time. In other words, if no food is obtained in a certain amount of time after a capture, a bird will leave the patch it is in and move to another. They further assumed, also implicitly, that having a giving-up time allows the birds to estimate their marginal capture rate, and that, in fact, the expected marginal capture rate is inversely proportional to the giving-up time. With these assumptions the predictions of optimal foraging theory become that an animal should have a constant giving-up time for all patch types within a habitat and that the giving-up time should be shorter in better habitats, where the average capture rate is higher (Krebs, Ryan, and Charnov, 1974). This is precisely what they found. Furthermore, predictions from the two "hunting-by-expectation" hypotheses differ from those of the optimal foraging hypothesis and are contradicted by the results.

The assumption that the birds adjust their giving-up times in such a way that they are inversely proportional to the marginal capture rates deserves further comment. This relationship is in the right direction, but until the statistical relationship between the two quantities is established it is not clear that the inverse of the giving-up time and the marginal capture rate should be proportional to one another. Empirically, however, that does appear to be true, for when the relative capture rates for four different habitats are compared with the relative reciprocals of the giving-up times for the four habitats, the similarity is quite amazing (2.04: 1.51: $1.34: 1.00$ vs. $2.07: 1.42: 1.37: 1.00)$. This is exactly what would be expected if these quantities were proportional to one another and if the birds do in fact adjust their marginal capture rates to be equal to the habitat average. Two of us (G.H.P. and E.L.C.) are at present investigating the statistical relationship between these two quantities.
The work of Orians (in press) illustrates well the complexity inherent in movement from one patch to another when the availability of food changes independently of the presence of the predator. For many years Orians has studied the social systems of marsh-nesting blackbirds (Icteridae) and has gathered many data relative to foraging. Food available to the birds was patchy in both space (e.g., waters' edge versus uplands) and time (emergence of aquatic insects was cyclical throughout the day and season); and the three bird species differed in their nest placement and efficiency of utilization of various patches. Orians found that the birds used many cues in their foraging and showed remarkable ability to "alter behavior as the resource base changed." He also studied several other aspects of foraging. His book should be consulted for a detailed discussion of the usefulness of the optimal foraging concepts in an uncontrolled field study.

\section{Optimal Patterns of Movements of Foraging Animals and Optimal Speed of Movement}

Movement patterns of foraging animals have received considerable attention from entomologists interested in the efficiency with which a parasite or predator finds its insect pest host (e.g., Laing, 1937, 1938; Varley, 1941; Flanders, 1947; Fleschner, 1950; Banks, 1954, 1957; Putnam, 1955; Wylie, 1958; Dixon, 1959; Kaddou, 1960; Mitchell, 1963; Bänsch, 1966; Chandler, 1969; Richerson and Borden, 1972), but has had comparatively little attention from evolutionary biologists (Turnbull, 1964; Tinbergen, Impekoven, and Franck, 1967; Croze, 1970; Cody, 1971, 1974; Janzen, 1971; Levin, Kerster, and Niedzlek, 1971; Pyke, 1974, in press a, b, unpub.; Smith, 1974a,b). The general finding of authors in the first group is that the animals they considered tend to "meander" until prey is encountered and then greatly increase their rate of turning, thereby tending to remain in the vicinity of the encountered prey (Laing, 1937, 1938; Flanders, 1947; Fleschner, 1950; Putnam, 1955; Banks, 1957; Dixon, 1959; Kaddou, 1960; Mitchell, 1963; Bänsch, 1966; Chandler, 1969; Croze, 1970; Richerson and Borden, 1972). That this behavior is an adaptation to feeding on prey which have a clumped distribution has been suggested by a number of these authors (Laing, 1937, 1938; 
Flanders, 1947; Dixon 1959; Kaddou, 1960; Mitchell, 1963). However, these studies leave many questions unanswered. For example, the extent to which an animal that is feeding on clumped prey should and does increase its rate of turning after encountering a prey is unknown, and the length of time over which the animal should and does maintain this change is also unknown. These and related problems have been considered by Cody $(1971,1974)$ and by Pyke (1974, in press-b).

Both of these authors have constructed simulation models of animal movements, have derived from them the properties of the optimal pattern of movements, and have compared these properties with available data. The simulation models of both authors are essentially identical and are constructed by supposing that an animal is moving from points on a uniform bounded grid to the nearest neighbors of these points and that food is concentrated at these points. This is equivalent to supposing that both the movements and the food have a discrete approximation and that the movements are restricted to a bounded foraging area. It is assumed that an animal moves according to four probabilities $\left(p_{S}, p_{R}, p_{L}\right.$, and $\left.p_{B}\right)$ assigned to the four directions - straight ahead, right, left and backwards - and that it moves independently of the presence of food at distant points. In other words, animals are assumed not to detect food at a distance by sight, smell, or other means, and hence not to alter their movements in response to such detection. Both Cody and Pyke argued that animals would be expected to exhibit patterns of movement which result in the maximum net rate of food intake and that this would occur if the frequency of path recrossing was minimized. They proceeded to determine the pattern of movements, characterized by the four probabilities, which would minimize this frequency of path recrossing (i.e., the optimal pattern of movements), and then compared these with observed patterns.

The number of possible combinations of the four probabilities $\mathrm{p}_{S}, p_{R}, p_{L}$, and $p_{B}$ is very large. It is possible, however, to choose these probabilities in such a way that they are biologically realistic and can be described by a single parameter. When animal movements are subdivided into segments and the changes in direction between successive segments are determined, it has been found, in almost all cases, that the frequency distributions are unimodal, bell-shaped distributions which have mean angles of approximately $0^{\circ}$ and which are truncated outside of the interval $(-\pi,+\pi)$ (Beukema, 1968; Siniff and Jessen, 1969; Kleerekoper et al., 1970; Cody, 1971; Levin, Kerster, and Niedzlek, 1971; Smith, 1971, 1974a,b; Pyke, 1974 , in press-b). The only exception to this is provided by data for the snowshoe hare which exhibit a bimodal distribution of changes in direction (Siniff and Jessen, 1969). Pyke has assumed that these distributions are all approximately truncated normal distributions on $(-\pi$, $+\pi$ ) with mean angles of $0^{\circ}$ and that each distribution is then uniquely determined by its variance. Upon this assumption, the probabilities (i.e.,p's) are determined by dividing the circular interval $(-\pi,+\pi)$ into four regions corresponding to straight ahead, to the right, etc., and setting the $p$ 's equal to the respective areas in these regions under the normal curve. Thus, the $p$ 's are chosen to be discrete approximations to the truncated normal distribution with different variances.

A convenient and useful way of describing the $p$ 's is to use the average vector component of successive movements in the straight-ahead direction. Pyke (1974), in press-a,b) used the term "directionality," of Levin, Kerster, and Niedzlek (1971) for this quantity. In the case of Pyke (1974, in press-a,b), "directionality" is simply $p_{S}-p_{B}$; it varies from 0 , when movement is random (i.e., $p_{S}=p_{R}=p_{L}=p_{B}=1 / 4$ ), to 1 , when movement is strictly in one straight line (i.e., $p_{S}=1, p_{R}=p_{L}=p_{B}=0$ ). If the distribution of changes in direction is a truncated normal one, then the directionality gives a unique representation of the distribution. It is also possible to think of movements in terms of the correlation between successive directions or the amount of turning. The greater the directionality, the larger is this correlation and the lower the amount of turning.

Cody $(1971,1974)$ has claimed that the movements of finch flocks in the Mohave Desert are optimal because they result in the lowest possible frequency of path recrossing. He simulated movements on a grid of $11 \times 11$ points with a reflecting boundary; i.e., when a boundary was hit, the next movement was assumed to be backwards. He found that with these restrictions the optimal directionality is very close to the observed directionality of the finch flocks, 
namely 0.64 (calculated as the average of $p(A)-$ $p(B) \equiv p_{S}-p_{B}$ from Table 2 of Cody, 1971). However, Pyke (1974, in press-b) has shown that optimal directionality depends on the size of the foraging area, as represented by the size of the grid, and also on the boundary behavior. Cody (1974) has claimed that the finch flocks were foraging in areas which were about $400 \mathrm{~m}$ $\times 400 \mathrm{~m}$ (i.e., $1236 \mathrm{ft} \times 1236 \mathrm{ft}$.). Since the average length of movement for the flocks was 182 feet (calculated from Table 2 of Cody, 1971), this foraging area of $1236 \mathrm{ft} \times 1236 \mathrm{ft}$ actually corresponds to a grid of size $7 \times 7$ points. For a reflecting boundary the optimal directionality for a grid of this size is 0.67 (Pyke, 1974 , in press-b), a value which is still quite close to the observed 0.64 . Hence, with a more appropriate grid size and a reflecting boundary, agreement between the predicted and observed values remains quite good.

Most animals, however, probably do not treat the boundaries of their foraging areas as reflecting boundaries. Just how do animals behave when they encounter a boundary? Pyke (1974, in press-b) observed the movements of broadtailed hummingbirds (Selasphorus platycercus) on a $6 \times 6$ grid of artificial flowers and found that, upon reaching a boundary of the grid, the birds moved according to probabilities $p^{\prime}{ }_{R}=0.46, p^{\prime}{ }_{L}$ $=0.42$ and $p^{\prime}{ }_{B}=0.12$; and when moving along a boundary the relevant probabilities were $p^{\prime \prime}{ }_{S}=$ $0.61, p^{\prime \prime}{ }_{R}$ or $p^{\prime \prime}{ }_{L}=0.22$, and $p^{\prime \prime}{ }_{B}=0.17$. These appear to be the only data at present available regarding movements of animals after hitting a boundary of their foraging area.

If the boundary behavior of the hummingbirds is typical of that for animals in general, then Pyke has shown that the simulation model in its present form would lead us to hypothesize that animals would always exhibit directionalities of their movements in the range 0.8 to 1.0 . For a realistic grid of $7 \times 7$ and the more realistic nonreflecting behavior of the hummingbirds, the optimal directionality for Cody's finch flock would become 0.80 , substantially different from the observed value of 0.64 . Pyke (1974, in press-b) has reviewed the literature on directionality of a variety of animals, including foxes, bees, butterflies, fish, and birds. The directionality for all of these studies ranges from 0.03 to 0.8 . Thus Pyke has found no overlap between the range of predicted values and the range of observed values.
There are two possible conclusions from this result. The first is that the hypothesis of optimal foraging is incorrect in this case. The second is that the assumptions of the model are incorrect. Pyke has argued that the assumption that animals move independently of the presence of food at distant points is probably incorrect for most animals and that most animals do in fact detect and respond to food at a distance. He finds support for this conclusion in a study of the movements of nectar-feeding bumblebees between inflorescences on plants that occur in large two-dimensional patches (Pyke, in pressa). He has formulated a mathematical model of the movements of bumblebees, a model which, in contrast to the assumption of the previous study, supposes that the movements are largely determined by visual responses to distant inflorescences. More precisely, the model supposes that a bumblebee, while at an inflorescence, chooses the next one to fly to by first aiming its departure in some direction relative to the direction of arrival at the present inflorescence. It then visually scans a sector about the aimed direction, and finally chooses the closest inflorescence within the sector. It is assumed that the bumblebee can vary the relationship between the aimed departure and arrival directions and the width of the scanned sector. These two parameters determine, in turn, properties of the distribution of changes in direction at the inflorescences and of the distribution of distances flown between inflorescences, and these properties are the output of the model. From the model Pyke has derived properties of the optimal pattern of movements (i.e., the pattern which results in the maximum net rate of energy intake) and has compared these with data on bumblebee movements. Though the predictions are qualitative only, agreement between them and the observations is encouraging.

The future of research in this area seems to be more uncertain than in other areas discussed above. Any future models of the foraging movements of an animal must surely include some approximation of the way in which animals use their senses to aid in detecting food. For this, of course, we need a quantitative knowledge of their sensory abilities and their use of them. Furthermore, to test the models the exact distribution of food will have to be determined, together with the relationship be- 
tween the cues used by the animals to detect food and the probability of detecting food, and with a knowledge of the distribution of the cues.

In the models of Cody and Pyke discussed above, the speed with which an animal moves was assumed to be fixed. Animals can certainly vary their speed of movement in both an immediate and an evolutionary sense, however. Hence, the following questions arise: what is the optimal speed of movement? and do animals move at this expected speed?

The problem of optimal speed of movement (in relation to energy intake) has been considered so far only by Ware (1975), in a study of planktivorous fish. Ware found that while the rate of gross caloric intake and the energetic cost of movement both increase with speed of movement, the net rate of caloric intake reaches a maximum at a speed which depends on the food density. Using data from Ivlev (1960), Ware showed that for this particular species of fish, the optimal speed of movement under Ivlev's experimental conditions was 111 meters per hour. The observed speed was 107 meters per hour, a value remarkably close to the expected. This is strong support for the optimal foraging hypothesis.

\section{THE FUTURE}

\section{A. Models Which Look at Several Factors at Once}

The approach used in this review follows the original approach used in the literature-to break the foraging process into several problems and consider each in isolation. Recently, several authors have begun to develop models which look at two or more aspects of foraging at the same time. For example, Orians and Pearson (in press) developed a model of "central place foraging" which they defined as occurring when an animal goes out from a central place (e.g., its nest), captures food, and then returns to the central place. Their models considered "where the animal should go" (choice of patches) and "what food items it should attack" (breadth of diet) at the same time. As expected, the predictions from their model resemble, but differ somewhat from, the predictions of the single-component models discussed above. At present, however, these new predictions remain untested.

A second example is provided by the work of
Taylor (unpub.) and Davidson (in prep.). They both consider the foraging by ants among patches of food placed at different distances from a colony. Within each patch there is a range of sizes of food particles. They then simultaneously consider the allocation of different numbers of workers to the different patches and the choice of particle size by individual workers within a patch. Taylor was able to predict qualitatively the foraging of both the colony as a whole and of the individual workers from an optimal foraging model.

As a third and final example we mention the work of McFarland and Sibly (1975). They have considered foraging as one of many activities to which an animal must allocate time, and their interest is in how an animal should and does allocate its time. From observations on how an animal (a dove) allocates its time in a wide range of conditions, they are able to determine the relative priorities of the animal for various activities and how these priorities change as a function of the "state" of the animal. They then use these priorities to successfully predict the behavior of the animal under new conditions.

\section{B. Information Gathering, Memory, and Estimation of Parameters}

As mentioned above, an implicit assumption of the models is that an animal "knows" or can estimate the magnitude of the parameters which appear in the equations. This means that an animal must be able to gather information relevant to these parameters, must "remember" this information at a later time, and must translate all the available information into estimates of the parameter values.

The ability of an animal to gather information must surely depend on the nature of its sensory apparatus and on physiological reactions which occur within the animal's body. These constraints immediately suggest the question: why do animals not possess sensory apparatuses which are better at gathering information than those they actually possess? We believe the answer to this question involves a trade-off between gains brought about by possession of more information and costs entailed in obtaining that information. As more and more information becomes available at a greater and greater cost, additional information is likely to be of relatively less and less value. For 
example, if it were advantageous for a bird to estimate the probability that a leaf chosen at random will have a food insect beneath it, a sample of 200 leaves would probably be little better than a sample of 100 . Just where a balance should be struck and where it is struck are problems for future research. The same kind of trade-off should occur with regard to an animal's "memory" (i.e., its ability to store and recall information), which should be a complicated function of the neural networks of an animal's brain. Progress in these areas of information-gathering and memory will undoubtedly depend on the development of some understanding of the magnitudes of gains and costs associated with changes in an animal's sensory system.

Perhaps the most rapid progress will occur in the areas of how animals translate information into estimates of parameters. This would seem to be expected from the fact that such familiar statistical notions as precision, power, and the like should provide unambiguous, quantifiable measures of the gains obtainable from the use of greater amounts of information, should suggest "optimal" statistics for animals thought to possess a certain amount of information, or, more generally, should suggest "optimal sampling schemes" for animals. As an example of this approach, let us consider once more the paper of Krebs, Ryan, and Charnov (1974). They have assumed implicitly that the only information used by their birds was a knowledge of the amount of time since the last capture of prey or if there had been no capture, the time since arrival in the patch. Using only this information there should be an optimal statistic that would estimate the expected instantaneous capture rate of the bird. If, as argued above, the expected, instantaneous capture rate is inversely proportional to the time since arrival or since the last capture, then the theory predicts that the birds should leave a patch when this time exceeds some threshold value. That is, the birds should employ a giving-up time. It is possible, however, that the birds also make use of a knowledge of the length of time between the last and the second-last capture. This knowledge would certainly allow the birds to estimate, using a different statistic, the expected instantaneous capture rate, with greater precision. If this increase in precision were potentially large, one would expect the birds to "invest" in the slight increase in memory and to use the optimal statistic for the total amount of information. It should be relatively easy, if sufficient data become available, to determine whether or not the birds actually use more information than the time since arrival or since the last capture; but this has not yet been done. In this case and in more general cases, the increases in precision should decline fairly quickly as more and more information is used, and hence one would expect that the memories of the birds would not extend back very far in time.

A beginning of research in this area of information gathering, memory, and parameter estimation has been made by two of us (G.H.P. and E.L.C.), while Bobisud and Potratz (1976) have considered a similar problem in the context of a model-mimic predation system. The latter authors conclude that a predator does better, in terms of "benefit per encounter," if it employs a single-trial learning scheme (i.e., short memory, little information used) than if it employs a multi-trial learning scheme (i.e., longer memory, more information). The assumptions of Bobisud and Potratz (1976) about possible kinds of multi-trial learning are rather restrictive, however, and consequently, more investigation of the problem is necessary before their conclusion can be considered to have generality.

\section{Coevolution}

In the future, optimal foraging theory and more general optimality theories should provide many insights into the nature of a variety of coevolutionary systems. Such coevolutionary systems might be of predators and their prey, competing species, symbiotic relationships, and the like (see, for example, Lawlor and Maynard Smith, 1976). To illustrate how all this might happen we shall briefly consider the coevolutionary system consisting of plants and the animals which pollinate them. In that system the animals obtain food (in the form of pollen or nectar) at the flowers of the plants, and in moving among the flowers they transfer pollen and thereby effect pollination of the plants.

The characters that should be coevolving in this system are, on the one hand, the pattern of movements of the animal pollinators among the flowers, and on the other hand, such plant 
characteristics as the number of flowers per plant, the spatial distribution of the flowers, and the amount of nectar per flower. Optimal foraging theory could make it possible to predict the movement behavior of the pollinators for a specified set of plant characteristics and also the way in which pollinators would be expected to change their behavior in response to changes in any of the characteristics of the plants. What is also needed in this case is an optimality theory that predicts, for a specified pattern of pollinator movements, the optimum characteristics of the plants. When the optimality theories for both the plants and the animals are combined, it should be possible to predict a "coevolutionary equilibrium" such that any changes in either the animals or the plants would result in departures from the optimum and hence would be selected against. This predicted equilibrium could then be compared with the actual situation in nature. One of us (Pyke) has attempted to carry out a coevolutionary study of this kind, but it leaves many questions unanswered (Pyke, unpub.; see also Covich, 1974). In general, such coevolutionary studies should be about as difficult as it is difficult to construct realistic optimality theories for the component species in the system. Nevertheless, we feel that these theories and any combinations of them will provide a much better understanding of the processes of evolution.

\section{Community Structure}

For many species, resources derived from foraging form the basis of population growth. The lack of resources available in food plus the loss of resources to higher trophic levels eventually cause population decline. Thus, predation can be the very substance of population regulation, as being a force that causes both increase and decrease in number and biomass (Huffaker, 1970). The foraging behavior of the individuals in a species determines the force of population increase, and the foraging behavior of that species' predators is a major force in the population decline of the species. The same foraging behaviors determine the trophic structure of a community and the similarity of the diets of the members of that community. Diet similarity is central to determination of competitive coexistence of species and thus to species diversity. Species diversity and trophic structure are essential ingredients of community stability. Thus predation, as determined by the foraging behavior of all animals in a community, is the core of community structure.

The role to be played by foraging theory in understanding communities will depend on the ability of the theory to predict trophic relationships. We already know that the theory can predict qualitatively certain aspects of individual foraging in simple environments. But the degree to which the theory can predict who eats what, when, and where will determine its usefulness in unraveling the mysteries of community structure. As food becomes scarce, individuals respond, as predicted by theory, by widening their choice of foods. If the individuals of two similar species occupying the same habitat both take a greater variety of food as food becomes scarce, their diets will tend to converge. Thus, as food becomes limiting, competition between the two species will become severe and one may displace the other. On the other hand, if a variety of habitat patches with different foods are available, patch utilization by the two species may converge or diverge depending on the similarity of their responses to declining food. The sum of patch and food choices determines the coexistence of the species. The only study we are aware of which uses optimal foraging theory (Optimal Diet) to predict overlap and then uses these to look at coexistence patterns is that of Werner (in press), in which three species of sunfish in southern Michigan lakes were studied. Laboratory experiments were used to determine the relevant parameters, such as handling times, for various prey types. The end-product was a set of utilization curves for the three species over a range of prey types. These were combined in measures of overlap, from which it was predicted that two of the species could coexist, while the "in-between" species could not invade. A survey of several lakes showed this to be indeed the case - the "in-between" species was absent from the habitat occupied by the other two. We find these results quite encouraging.

A number of qualitative predictions of foraging theory can also help us understand community structure, regardless of the ability of the theory to predict details. For example, we may compare two communities with the same diversity of resources at one trophic level but with differences in the degree to which the production is cyclical even though total production is 
similar. How might the diversity of predators on the resources in these two communities differ? The number of species in each community will depend on the specialization of the predators and their degree of niche overlap. The possibilities for specialization are reduced in the cyclical community. As resources decline, each species must respond by broadening the range of acceptable types of resources; this relationship increases niche overlap and prevents species from specializing on individual types of resource. The net result predicts that the cyclical community will have fewer species, a prediction which may be helpful in interpreting latitudinal gradients in diversity.

\section{E. Optimality Thinking in Population Biology}

The usefulness of a point of view should be measured by the insight it provides. We suggest that the optimality viewpoint of predation has proved itself quite useful. It has suggested testable hypothese which would not have been considered under more traditional viewpoints, and furthermore these hypotheses have received quite strong support from the data so far collected.

The present clan of models will surely prove to be too simplistic, but they seem to be a necesary beginning. Only time will tell whether this beginning will die in its embryonic stages or whether it will grow to maturity. The present authors are very optimistic about the future of optimal foraging theory and feel that, as it grows, it will continue to provide a predictive understanding of how animals forage and insights into many related areas of biology.

\section{ACKNOWLEDGMENTS}

We have benefited from the critical advice of many colleagues. Nolan Pearson, J. R. Krebs, G. H. Orians, T. W. Schoener, E. E. Werner, J. Emlen, and E. Pianka have been particularly helpful. E.L.C. wishes to thank R. Dawkins and J. M. Cullen for spirited discussion, and G. H. Orians and J. R. Krebs for several years of fruitful give and take. G. H. P. wishes to thank J. Pulliam for helping to make his many visits to Tucson so enjoyable.

\section{LIST OF LITERATURE}

Altmann, S. A., and S. S. WAgner. In press. A general model of optimal diet. In VI. Congress of the Intermational Primatological Society.

ANDREWS, R. M. 1971. Food resource utilization in some tropical lizards. Ph.D. Thesis, U. of Kansas.

Banks, C. J. 1954. The searching behaviour of coccinellid larvae. Anim. Behav., 2: 37-38.

- 1957. The behaviour of individual coccinellid larvae on plants. Br. J. Anim. Behav., 1: 12-24.

BÄNSCH, R: 1966. On prey-seeking behaviour of aphidophagous insects. In Hodek, I. (ed.), Ecology of Aphidophagous Insects, p. 123-128. Academia, Prague.

Beukema, J. J. 1968. Predation by the three-spined stickleback (Gasterosteus aculeatus): the influence of hunger and experience. Behaviour, 31: 1-126.

Bobisud, L. E., and C. J. Potratz. 1976. One-trial versus multi-trial learning for a predator encountering a model-mimic system. Am. Nat., $110 ; 121$ 128.

Chanderr, A. E. F. 1969. Locomotory behaviour of first instar larvae of aphidophagous Syrphidae (Diptera) after contact with aphids. Anim. Behav., 17: 673-678.

Charnov, E. L. 1973. Optimal foraging; some theoretical explorations. Ph.D. Thesis, University of Wash.

- 1976a. Optimal foraging; attack strategy of a mantid. Am. Nat., 110: 141-151.
- 1976b. Optimal foraging; the marginal value theorem. Theor. Pop. Biol., 9: 129-136.

Charnov, E. L., G. H. Orians, and K. Hyatt. 1976. Ecological implications of resource depression. Am. Nat., 110: 247-259.

Cody, M. L. 1971. Finch flocks in the Mohave Desert. Theor. Pop. Biol., 2: 142-158.

- 1974. Optimization in ecology. Science, 183: 1156-1164.

Соoк, R. M., and S. S. HubBard. In press. Adaptive searching strategies in insect parasites. J. Anim. Ecol.

Covich, A. 1972. Ecological economics of seed consumption by Peromyscus; a graphical model of resource substitution. Conn. Acad. Arts \& Sci., 44: 69-94.

-1974. Ecological economics of foraging among coevolving animals and plants. Ann. Missouri Bot. Gardens., 61: 794-805.

- 1976. Analyzing shapes of foraging areas: some ecological and economic theories. Ann. Rev. Ecol. Syst., 7: 235-258.

Croze, H. 1970. Searching image in carrion crows. Hunting strategy in a predator and some antipredator devices in camouflaged prey. Z. Tierpsychol., 5: 1-86.

DeBenedictus, P. A., F. B. Gill, F. R. Hainsworth, G. H. Pyke, and L. L. Wolf. In press. Optimal meal size in hummingbirds. $A m$. Nat. 
Dixon, A. F. G. 1959. An experimental study of the searching behaviour of the predatory coccinellid beetle Adalia decempunctata (L.) J. Anim. Ecol., 28: 259-281.

EgGers, D. M. 1975. A synthesis of the feeding behavior and growth of juvenile sockeye salmon in the limnetic environment. Ph.D. Thesis, Univ. of Wash.

EmLen, J. M. 1966. The role of time and energy in food preference. Am. Nat., 100: 611-617.

—. 1973. Ecology: An Evolutionary Approach. Addison-Wesley, Reading (Mass.).

Emlen, J. M. and M. G. R. EmLen. 1975. Optimal choice in diet: test of a hypothesis. Am. Nat., 109: 427-435.

Estabrook, G. F., and A. E. Dunham. 1976. Optimal diet as a function of absolute abundance, relative abundance, and relative value of available prey. Am. Nat., 110: 401-413.

Flanders, S. E. 1947. Elements of host discovery exemplified by parasitic hymenoptera. Ecology, 28: 299-309.

Fleschner, C. A. 1950. Studies on searching capacity of the larvae of three predators of the citrus red mite. Hilgardia, 20: 233-265.

GibB, J. A. 1958. Predation by tits and squirrels on the eucosmid Ernarmonia conicolana (Heyl.) J. Anim. Ecol., 27: 275-296.

- 1960. Populations of tits and goldcrests and their food supply in pine plantations. Ibis, 102: 163-208.

Gill, F. B., and L. L. Wolf. 1975a. Foraging strategies and energetics of East African sunbirds at mistletoe flowers. Am. Nat., 109: 491-510.

- $1975 \mathrm{~b}$. Economics of feeding territoriality in the golden-winged sunbird. Ecology, 56: 333-345.

Goss Custard, J. D. In press. Optimal foraging and the size selection of worms by redshank (Tringa totanus). Anim. Behav.

Huffaker, C. B. 1970. The phenomenon of predation and its roles in nature. Proc. Adv. Study Inst. Dynamics, Nos. Pop. (Oosterbeek), 1970; 327-343.

IVLEv, V. S. 1961. Experimental Ecology of the Feeding of Fishes. Yale Univ. Press, New Haven.

JanzeN, D. H. 1971. Englossine bees as long-distance pollinators of tropical plants. Science, 171: 203205.

Janzen, D. H., and W. J. Freeland. 1974. Strategies in herbivory by mammals: the role of plant secondary compounds. Am. Nat., 108: 269-289.

Kaddou, I. 1960. The feeding behaviour of Hippodamia quinquesignata (Kirby) larvae. Univ. Calif. Publs. Ent., 16: 181-232.

KATZ, P. L. 1974. A long-term approach to foraging optimization. Am. Nat., 108: 758-782.

KEAR, J. 1962. Food selection in finches with special references to interspecific differences. Proc. Zool. Soc. London, 138: 163-204.

KleEREKoper, H., A. M. Timms, G. F. Westlake, F. B.
Davy, T. Malar, and V. M. Anderson. 1970. An analysis of locomotor behaviour of goldfish (Carassius auratus). Anim. Behav., 18: 317-330.

KREBS, J. R. 1974. Behavioral aspects of predation. In P. P. Bateson and P. H. Klopfer (eds.), Perspectives in Ethology, p. 73-111. Plenum Press, N. Y.

Krebs, J. R., J. Ryan, and E. L. Charnov. 1974. Hunting by expectation or optimal foraging? A study of patch use by chickadees. Anim. Behav., 22: 953-964.

Krebs, J. R., T. Ericksen, M. I. Webber and E. L. ChaRnov. In press. Optimal prey selection in the Great Tit (Parus major). Anim. Behav.

LaCK, D., and D. F. Owen. 1955. The food of the swift. J. Anim. Ecol., 24: 120-136.

LAING, J. 1937. Host finding of insect parasites. I. Observations on the finding of hosts by Alysia manducator, Mormoniella vitripennis and Trichogramma evanescens. J. Anim. Ecol., 6: 298-317. 1938. Hosting finding by insect parasites. II. The chance of Trichogramma evanescens finding its hosts. J. Exp. Biol., 15: 281-302.

Lawlor, R., and J. Maynard Smith. 1976. The coevolution and stability of competing species. Am. Nat., 110: 79-99.

Levin, D. A., H. W. Kerster, and M. Niedzlek. 1971. Pollinator flight directionality and its effect on pollen flow. Evolution, 25: 113-118.

MACARThUR, R. H. 1972. Geographical Ecology. Harper and Row, New York.

MacArthur, R. H., and E. R. Pianka. 1966. On optimal use of a patchy environment. Am. Nat., 100: 603-9.

MacArthur, R. H., and E. O. Wilson. 1967. The Theory of Island Biogeography. Princeton Univ. Press, Princeton.

Maynard Smith, J. 1974. Models in Ecology. Cambridge Univ. Press, Cambridge.

McFarland, D. J., and R. M. Sibly. 1975. The behavioural final common path. Phil. Trans. Roy. Soc. Lond. (B), 270: 265-293.

Menge, J. L., and B. A. Menge. 1974. Role of resource allocation, aggression and spatial heterogeneity in coexistence of two competing intertidal starfish. Ecol. Monogr., 44: 189-209.

Mitchell, B. 1963. Ecology of two carabid beetles, Bembidion lampros (Herbst) and Trechus quadristriatus (Schrank). I. Life cycles and feeding behaviour. J. Anim. Ecol., 32: 289-299.

Orians, G. H. In press. The Adaptations of Marsh Nesting Blackbirds. Princeton Univ. Press, Princeton.

Orians, G. H., and N. E. Pearson. In press. On the theory of central place foraging. In D. F. Horn (ed.), Analysis of Ecological Systems, Ohio State University Press, Columbus.

Pearson, N. E. 1974. Optimal foraging theory. Quan. Science paper \# 39; Center for Quan. Science in Forestry, Fisheries and Wildlife, Univ. of Washington, Seattle. 
- 1976. Optimal foraging: some theoretical consequences of different feeding strategies. Ph.D. Thesis, Univ. of Washington.

Pulliam, H. R. 1974. On the theory of optimal diets. Am. Nat., 108: 59-74.

- 1975. Diet optimization with nutrient constraints. Am. Nat., 109: 765-768.

Putnam, W. L. 1955. Bionomics of Stethorus punctillum Weise (Coleoptera: Coccinellidae) in Ontario. Can. Entomol., 87: 9-33.

Pyкe, G. H. 1974. Studies in the foraging efficiency of animals. Ph.D. Thesis, Univ. of Chicago.

- In press, a. Optimal foraging: movement patterns of bumblebees. Theoret. Pop. Biol.

- In press, b. Are animals efficient harvesters? Anim. Behav.

RAPPORT, D. J. 1971. An optimization model of food selection. Am. Nat., 105: 575-87.

Rapport, D. J., and J. E. Turner. 1977. Economic models in ecology. Science, 195: 367-373.

Richerson, J. V., and Borden, J. H. 1972. Hostfinding behavior of Coeloides brunneri (Hymenoptera: Braconidae). Can. Entomol., 104: 1235-1250.

Root, R. B. 1967. The niche exploitation pattern of the Blue-gray Gnatcatcher. Ecol. Monogr., 37: 317-350.

Royama, T. 1970. Factors governing the hunting behavior and selection of food by the Great Tit, Parus major. J. Anim. Ecol., 39: 619-668.

- 1971. A comparative study of models for predation and parasitism. Res. Pop. Ecol., Suppl. 1: $1-91$.

Schoener, T. W. 1969. Models of optimal size for a solitary predator. Am. Nat., 103: 277-313.

- 1971 . Theory of feeding strategies. Ann. Rev. Ecol. Syst., 11: 369-404.

- 1974 . The compression hypothesis and temporal resource partitioning. Proc. Nat. Acad. Sci., 71: 4169-4172.

Schoener, T. W., and D. H. Janzen. 1968. Notes on environmental determinants of tropical vs. temperate insect size patterns. Am. Nat., 102: 207-224.

Sexton, O. J., J. Baumen, and E. Owen. 1972. Seasonal food habits of Anolis limifrons. Ecology, 53: 182-186.

Siniff, D. B., and C. R. Jessen. 1969. A simulation model of animal movement patterns. In J. B. Cragg (ed.), Advances in Ecological Research, Vol. 6, p. 185-219. Academic Press, New York.

Smith, C. C. 1970. The coevolution of pine squirrels (Tamiasciarus) and conifers. Ecol. Monogr., 40: 349-371.

Smith, J. N. M. 1971. Studies of the searching behaviour and prey recognition of certain vertebrate predators. D. Phil. Thesis, Oxford University. 1974a. The food searching behaviour of two European thrushes: I. Description and analysis of search paths. Behaviour, 48: 276-302.

1974b. The food searching behaviour of two European thrushes. II. The adaptiveness of the search patterns. Behaviour, 49: 1-61.

Smith, J. N. M., and R. Dawkins. 1971. The hunting behavior of individual Great Tits in relation to spatial variations in their food density. Anim. Behav., 19: 695-706.

Thompson, W. A., I. Vertinsky, and J. R. Krebs. 1974. The survival value of flocking in birds: a simulation model. J. Anim. Ecol., 43: 785-820.

Timin, M. E. 1973. A multi-species consumption model. Math. Biosciences, 16: 59-66.

Tinbergen, N., M. Impekoven, and D. Franck. 1967. An experiment on spacing-out as a defense against predation. Behaviour, 28: 307-320.

Tullock, G. 1970. The coal tit as a careful shopper. Am. Nat., 105: 77-80.

Turnbull, A. L. 1964. The search for prey by a web-building spider Achaearanea tepidariorum (C. L. Koch) (Araneae, Theridiidae). Can. Entomol., 96: 568-579.

VARLEY, G. C. 1941. On the search for hosts and the egg distribution of some chalcid parasites of the knapweed gall-fly. Parasitology, 33: 47-66.

WARD, P. 1965. Feeding ecology of the black-faced Dioch Quelea quelea in Nigeria. Ibis, 107: 173-214.

WARE, D. 1971. The predatory behavior of rainbow trout. Ph.D. Thesis, Univ, of British Columbia.

- 1975. Growth, metabolism and optimal swimming speed in a pelagic fish. J. Fish Res. Bd. Can., 32: $33-41$.

Werner, E. E. 1974. The fish size, prey size, handling time relation in several sunfish and some implications. J. Fish. Res. Bd. Can., 31: 153-156.

- In press. Species packing and niche complementarity in three sunfishes. Am. Nat.

Werner, E. E., and D. J. Hall. 1974. Optimal foraging and size selection of prey by the bluegill sunfish (Lepomis mochrochirus). Ecology, 55: 10421052.

Westoby, M. 1974. An analysis of diet selection by large generalist herbivores. Am. Nat., 108: 290304.

Wilson, D. S. In press. Deducing the energy ayailable in the environment from stomach contents. Biotropica.

Wolf, L. L. 1975. Energy intake and expenditures in a nectar-feeding sunbird. Ecology, 56: 92-104.

Wolf, L. L., F. R. Hainsworth, and F. B. Gill. 1975. Foraging efficiencies and time budgets in nectar-feeding birds. Ecology, 56: 117-128.

Wylie, H. G. 1958. Factors that affect host finding by Nasonia vitripennis (Walk.) (Hymenoptera: Pteromalidae). Can. Entomol., 90: 597-608. 